\title{
LXIV Open Seminar on Acoustics OSA 2017 Piekary Śląskie, Poland, September 11-15, 2017
}

\author{
Abstracts \\ Comparing Audio Coders in Voip Technology \\ Aleksiejuk Mikołaj, Betkier Daniel \\ University of Finance and Management \\ Pawia 55, 01-030 Warszawa, Poland
}

The raising quality of conversations is a result of the implementation of innovative solutions used in voice transmission in telecommunication networks. One, out of a significant number, of such solutions is the ever-changing encryption technique. It is the choice of suitable parameters of encoders and decoders that guarantees high performance of voice connections. It is the choice of suitable parameters of encoders and decoders that guarantees high performance of voice connections. The main aim of this research was to select the codec with the highest effectiveness in this particular service.

\section{Dimensional Analysis for the Model Tests} of Sound Absorption by Perforated Panels

\section{BARUch Katarzyna, KAmisiński Tadeusz}

Department of Mechanics and Vibroacoustics

AGH University of Science and Technology

al. Mickiewicza 30, 30-059 Kraków, Poland

Scaled modelling is very common in the areas of science such as aerodynamics or fluid mechanics, since defining characteristic numbers enables to determine relations between objects under test/investigation and their models. In acoustics, scaled modelling is aimed mainly at investigation of room acoustics and sound absorption phenomenon. Despite such a range of application, there is no method developed that would enable scaling acoustical perforated panels freely, maintaining their sound absorption coefficient. The paper discusses a number of theoretical and numerical analyses performed in order to suggest elementary relations enabling scaling of acoustical perforated panels. Given relations, together with properly designed measurement and calculation procedures, will allow to produce measurement samples equivalent to full-scale acoustical panels. Obtained results will allow to make the process of designing acoustical perforated panels easier and the costs of producing prototypes - lower. Furthermore, it will also be possible to simulate sound absorption characteristics of perfo- rated panels applied in the models of existing or designed interiors.

$$
\star \star \star
$$

Adaptation and Preliminary Evaluation of Phong Reflection Model in Room Acoustic Simulations

BineK Wojciech, KAMisiński Tadeusz

Department of Mechanics and Vibroacoustics AGH University of Science and Technology al. Mickiewicza 30, 30-059 Kraków, Poland

Room Acoustics Rendering Equation provides a mathematical background for description of geometrical acoustics algorithms. It requires a knowledge of Bidirectional Reflectance Distribution Function that describes materials reflective properties. Commonly used reflection model combining Lambert scattering and ideally specular reflection can be applied but it is far from real material behaviour. We present an adaptation of Phong reflection model, used in computer graphics, for acoustics simulations. A Phong model was fitted to measured reflection distributions and evaluated in ray tracing simulations. Our approach doesn't introduce any new material parameters as they can be derived with acceptable loss of information from commonly used scattering coefficient thus it can be used as direct replacement for current reflection model. Using Phong reflection model provides better reflection distribution approximation than combined Lambert scattering and ideally specular reflection.

$$
\star \star \star
$$

\section{Noise Reduction from Industrial Installations} - Steam Silencers

\author{
BogusŁaWski Grzegorz ${ }^{1}$, Kopania Joanna ${ }^{1}$ \\ KARCZEWSKI Jacek ${ }^{2}$ \\ ${ }^{1}$ Lodz University of Technology \\ Piotrkowska 266, 90-924 Łódź, Poland \\ ${ }^{2}$ Institute of Power Engineering \\ OTC Thermal Technology Branch "ITC" \\ Dąbrowskiego 133, 93-208 Łódź, Poland
}

In industrial installations economically viable energy carrier is a steam with high operating parameters like pressure, temperature or mass volume. Heat treatment technology using steam is indispensable in many industries. However, the practical use of steam in installations requires the 
specific safety features such as reducing stations, cooling units, fire extinguishers, relief valves, safety valves or starting valves. Valves allow you to dump the system into the vapor atmosphere, which expands to atmospheric pressure at the outlet of the discharge pipeline. This phenomenon is most often accompanied by noise above $140 \mathrm{~dB}$, what is very troublesome for people and the environment. This article discusses the issue of noise emission during steam discharge from safety or starting valves to the atmosphere. Also discussed are the ways to reduce it with the help of steam silencers.

\section{Sample Material for Speech Quality Measurements in DAB + Broadcasting}

\section{BraChMaŃSKI Stefan}

Faculty of Electronics

Wrocław University of Science and Technology

Wybrzeże Wyspiańskiego 27, 50-370 Wrocław, Poland

The final phase of new $\mathrm{DAB}+$ radio stations realization demands carrying out of many tests, including the quality ones. The speech quality measurements are carried out according to the quality and intelligibility criteria. In the presented paper the test sets for speech quality evaluation for both criteria are introduced. The sets were prepared for demands of the new local Wroclaw DAB + station. The issues related with realization of the new station are presented together with speech quality measurements according to intelligibility and quality criteria. In the first case this is the speech intelligibility measure and in the second Absolute Category Rating (ACR) which gives the result in five grades scale MOS (Mean Opinion Score) evaluation.

$$
\star \star \star
$$

An Influence of the Number of Reflected Modules and the Frequency on the Efficiency of the Muffler with the Directed Energy

Brański Adam ${ }^{1}$, PręDKA Edyta ${ }^{1}$, BŁĄD Grzegorz ${ }^{2}$

${ }^{1}$ Department of Acoustics

${ }^{2}$ Department of Electronic and Telecommunications Systems

Faculty of Electrical and Computer Engineering Rzeszów University of Technology

al. Powstańców Warszawy 12, 35-959 Rzeszów, Poland

Up to now, internal partitions make simple elements which provide additional sound wave reflections. As a result, a part of the sound wave turns around toward the source or back and forth inside the muffler. This effect prevents some sound energy transmission past the muffler. The number, shape and configuration of partitions remain a challenging task.

In the paper a new two partitions element is proposed and they together are named as the Reflected Module (ReM). The ReM directs the sound energy at exactly determined place of the muffler domain. This assures a better attenuation of the sound. An influence of the number of the fixed shape ReM and the frequency of the sound on the efficiency of the muffler is examined in experimental manner. It has been demonstrated that in some range of the frequency, adding successive ReM, the efficiency is become better and better. Nevertheless, in the future, it is proposed an optimization of some ReM parameters.

$$
\star \star \star
$$

The Influence of Baffle System Layout on Acoustic Absorption of the Structure

\section{Brawata Krzysztof, Baruch Katarzyna KAMisiński Tadeusz}

Department of Mechanics and Vibroacoustics AGH University of Science and Technology al. Mickiewicza 30, 30-059 Kraków, Poland

Baffle-type acoustic systems are absorbing spatial elements assembled particularly in spaces with no possibility of suspended ceiling installation. Such systems can be encountered both in public buildings (e.g. halls, atria, sports halls), as well as industrial sites. Baffle-type systems are usually hung in rows. The manufacturers of these systems most often provide the values of acoustic absorption for a few standard distances between them. These values are obtained only by means of measurements. If the distances between the rows are different or the systems are placed in a different configuration (e.g. chessboard or circle), it is necessary to conduct measurements of a given layout in a reverberation chamber. The present paper presents the analysis of the influence of a geometrical layout of baffletype systems on the acoustic absorption of the whole system. The study was conducted with the employment of 1:8 scale models and CATT-Acoustic software. The obtained results point to the layouts with more favourable acoustic parameters.

\section{Using Statistical Methods for Evaluation of Sound Field Diffusion - Experimental Verification}

Chojnacki Bartłomiej, Pilch Adam, Kamisiński Tadeusz

Department of Mechanics and Vibroacoustics

AGH University of Science and Technology

al. Mickiewicza 30, 30-059 Kraków, Poland

It is very important for the acoustician to know the level of sound field diffusion of a room at the moment of designing it. This parameter is not relevant for laboratory rooms - like reverberation chambers only, but may also describe very well sound field in rooms with qualified acoustics required. Parameters developed so far use complicated algorithms which process sound reflections coming from different directions or the results of sound intensity measurements. Both approaches force using specialized equipment. This paper discusses the methods for evaluation of sound field diffusion based on popular statistical methods, such as kurtosis of normalized impulse response or standard deviation of the reverberation time. The authors performed measurements of a chosen auditory room at the Department of Mechanics and Vibroacoustics AGH in two variants. Based on the recorded impulse responses of the room the parameters suggested by the authors were calculated and the level of sound field diffusion was evaluated. 
Distribution Functions and Time Constants of the Process of Cell Concentration Changes in Bodily Fluids in the Field of Ultrasonic Standing Wave

\section{Czyż Henryka, JAsińsKi Tadeusz \\ WŁOCH Andrzej}

The Faculty of Mathematics and Applied Physics

Rzeszow University of Technology

al. Powstańców Warszawy 8, 35-959 Rzeszów, Poland

This work deals with the problem of the interaction of acoustic waves with dispersions. This interaction can be used for separating the dispersed particles from the fluid. The aim of this work is to make a critical analysis of the equation of red blood cell motion in plasma in the field of forces occurring in ultra-sonic standing wave. The paper contains transformations of the equation taking into account different types of forces arising from the acoustic field and force of resistance.

The general properties of the solutions of motion equation have been given. The solutions for the parameters of the acoustic field and blood cells which are interesting in term of practical applications have been discussed. The time constant of the growth was estimated and the formulas, which determinate the time needed to obtain the assumed concentration increase, have been derived.

\section{Acoustic Investigations of Ionic Liquids, Structure-Property Relationship and Applications}

\section{DzIDA Marzena}

Institute of Chemistry

Silesian University

Szkolna 9, 40-006 Katowice, Poland

Ionic liquids (ILs) are one of the most promising class of substances, which characterize low volatility, a broad range of the liquid state, and high thermal stability. ILs have many industrial applications, including synthesis, separation, and biomass conversion. ILs can be used as hydraulic fluids, industrial lubricants, and heat transfer media. The influence of temperature and/or pressure on industrial processes and on the design of engineering devices, for which ILs could be used on a commercial scale, is still in the spotlight. Novel applications in industry require knowledge of thermophysical properties of ILs. The speed of sound is one of the key quantities for the equation of state development. The acoustic method is a commonly accepted computational procedure to determine $\mathrm{pVT}$ data as well as the isobaric and isochoric heat capacity, isobaric expansibility, isentropic and isothermal compressibility. Since the thermodynamic speed of sound is required. However, many ILs are generally much more viscous than conventional molecular organic liquids and exhibit dispersive effects at relatively low frequency range. Thus, the conditions allow to obtain the thermodynamic speed of sound which can be used to determine relevant quantities and to study the relation to IL structures will be presented and discussed.

\section{Designing of Public Address Systems on Railway Platforms}

\section{Dziechciński Paweł, Hadyk Maciej}

Chair of Acoustics and Multimedia

Wrocław University of Science and Technology

Wybrzeże Wyspiańskiego 27, 50-370 Wrocław, Poland

The legislation currently in force in the European Union, require that spoken information transmitted by the public address system of a railway station shall have a minimum STIPA value of 0.45 . The results of analyses, which should provide useful guidance for designers of public address systems are presented. Analyses were limited to the open platforms, where speech transmission index mainly depends on signal-to-noise ratio. The minimum values of signal-to-noise ratio to meet the requirements under typical acoustic conditions on the platform are presented. Maximum distance between loudspeakers, for which there is no danger of reduction of speech intelligibility as a result of the unfavourable relationship between delays and signal levels is also calculated. Attention has also been paid to the uniformity of coverage, which is not a subject of formal requirements, but has a significant impact on passenger comfort and is a part of good engineering practice.

$$
\star \star \star
$$

\section{An Application of Ultrasounds to Study the Properties of Wood}

Engel Marcin, Gudra Tadeusz

Wrocław University of Science and Technology Wybrzeże Wyspiańskiego 27, 50-370 Wrocław, Poland

Wood is a strongly anisotropic material. Its properties depend on the anatomical directions in the tree trunk. In the paper the authors presented the results of longitudinal and transverse wave velocity measurements for different species of wood: acacia, birch, oak, Italian poplar, black poplar. Ultrasonic measurements give an opportunity for qualitative classification (strength) of the wood. Most properties of wood are not dependent on moistness, which above a certain limit value affects only slightly the propagation velocity of the ultrasonic wave; the velocity can be used as criterion for assessing the quality of wood drying. Modulus of elasticity, calculated on the basis of velocity and density measurements, is a sensitive parameter, responsive to desorption of wood splitting. Both parameters: the velocity of the ultrasound wave propagation and the attenuation coefficient can be used to assess the degree of degeneration of wood tissue.

\section{Identification of Distortions Introduced During the Measurements of a Dynamic Loudspeaker Thiele-Small Parameters}

\section{Flach Artur, Chojnacki Bartłomiej}

Department of Mechanics and Vibroacoustics AGH University of Science and Technology al. Mickiewicza 30, 30-059 Kraków, Poland

Knowing Thiele-Small parameters is essential at the moment of designing the enclosure of a loudspeaker. 
PN-EN 60268-5 standard, which describes the measurement procedure, does not contain many details influencing the accuracy of the results. This factors, among others, are: placing the loudspeaker during the measurement, background level or magnetic interference. The presence of certain objects may also cause problems by changing the value of magnetic induction of the loudspeaker or electrical parameters of the coil. This paper discusses the results of the measurements conducted in different conditions simulating various types of interferences and distortions and preliminarily identifies their impact on particular T-S parameters.

Effective Analysis

of Selected Pitch Detection Methods

in Singing Voices Assessments

\section{GAwLIK Mateusz}

WszoŁeK Wiesław

Department of Mechanics and Vibroacoustics

AGH University of Science and Technology

al. Mickiewicza 30, 30-059 Kraków, Poland

Voice analysing problems, presented in the literature, concern mainly recognition, identification, verification or diagnostics of speakers. Authors focus only on speech surveys. Unfortunately, a range of studies of singing was not the subject of great interest by scientists. Results from presented research show that the most elementary parameter used in voice analysing is fundamental frequency. In this paper, we present pitch detection methods of singing signal in hope of choosing the best one. We decided to compare three common known and one less popular detection methods: zero crossing analysis (ZCA), cepstrum analyse, autocorrelation function $(\mathrm{ACF})$ and maximal amplitude analyse $(\mathrm{PR})$. The first conclusion from series of experiments is necessity of choosing probes to analyse in the proper way, because singing voice can change its basic frequency period by period approximately by two octaves. This paper presents a precise analysis of the pitch detection by selected methods in singing voices analysis.

\section{Measurement of Insertion Loss of the Acoustic Reflective Muffler for the Single Mode Excitation}

GorazD Łukasz, Kolber Karolina SnAKowska Anna, JuRkiewiCz Jerzy

Department of Mechanics and Vibroacoustics AGH University of Science and Technology al. Mickiewicza 30, 30-059 Kraków, Poland

The paper presents the results of experimental investigations of the insertion loss (IL) of two reflective mufflers for the reduced frequency (Helmholtz number) above the so-called plane wave approximation, which have been applied by the vast majority of theoretical and experimental studies. The studied mufflers differed in the size of the air gap and were mounted in a cylindrical waveguide provided with anechoic terminations and so corresponding to the theoretical model of an infinite waveguide. In order to better determine how the higher Bessel radial and circumferential modes are attenuated, the first three modes have been generated for the same reduced frequency $k a=3.5$ using the mode synthesizer in the form of a rotary pointsource matrix.

Due to the waveform decomposition on individual modes, their relative amplitudes were determined both in the reference channel and after passing through the muffler, which allowed to calculate the attenuation for each of them. Propagation of a single-mode wave greatly reduces the complexity of the acoustic field description and its analysis. The results obtained can be used to verify other test methods. Incorporation of higher modes in the carried-out analysis is extremely important, as the often considered approximation of the low frequency limit assuming only the plane wave propagation can lead to significant errors in determining the attenuation effect.

$$
\star \star \star
$$

\section{Application of Modified Prony Method to Bottom and Object Detection}

Grall Piotr

MARsZAL Jacek

Department of Marine Electronic Systems Faculty of Electronics, Telecommunications and Informatics

Gdansk University of Technology

Narutowicza 11/12, 80-233 Gdańsk, Poland

The article presents application of the new (proposed by the authors) modification of Prony method to determine direction-of-arrival of acoustic wave reflected from the sea bottom and bottom objects. Application of modified Prony method allows accurate determination of multiple sources of acoustic reverberation, thus allowing unimpeded operation in multipath environment. Utilized simulations represent typical, generic configuration of receiver, bottom, sea surface and bottom. Accuracy of depth determination is assessed for its compliance with the standards published by International Hydrographic

Organization. Object detection capabilities are assessed as well. The article ends with general conclusions regarding suitability of modified Prony method to hydrographic applications.

\section{Interesting Issues about the Vibration Isolation of Buildings and Floors}

\section{HoRVÁTH Zoltán}

Department of Programming Languages and Compilers Faculty of Informatics Eötvös Loránd University Budapest, Hungary

A few interesting issues are discussed in the presentation that may not be the first points of consideration during the design process, but have an influence on the performance of the vibration isolation solution. 
Effect of Magnetite Nanoparticles on Ultrasonic and Magnetic Hyperthermia

KACZMAREK Katarzyna ${ }^{1}$, HoRnowski Tomasz ${ }^{1}$

BIELAS Rafał ${ }^{1}, \dot{Z}_{\text {AK }}$ Dorota $^{1}$

Timko Milan ${ }^{2}$, JÓzEFCZAK Arkadiusz $^{1}$

${ }^{1}$ Institute of Acoustics, Faculty of Physics

Adam Mickiewicz University

Umultowska 85, 61-614 Poznań, Poland

${ }^{2}$ Institute of Experimental Physics

Slovak Academy of Sciences

Watsonova 47, 04001 Košice, Slovakia

Hyperthermia is a very promising anti-cancer medical treatment. It induces heat in cancer tissues which leads to their weakening. Weaken cells are therefore more susceptible for other cancer therapies like radiotherapy or chemotherapy. Heat can be induced by means of ultrasonic waves and alternating magnetic field. Magnetic hyperthermia uses superparamagnetic iron oxide nanoparticles heated by an externally applied AC magnetic field. Magnetic particles can also be used as sonosensitizers in ultrasonic hyperthermia. The obtained experimental results have shown that the addition of sonosensitizers enhances heat production by ultrasound wave in the tissuemimicking phantom due to increase of ultrasound attenuation. Thus the presence of magnetite nanoparticles in the phantoms leads to shorter duration time of hyperthermia treatments and reduced intensity of ultrasonic wave required to obtain clinical effect.

This work was supported by a Polish National Science Centre grant, no DEC-2015/17/B/ST7/03566.

$$
\star \star \star
$$

The Effect of Magnetic Nanoparticles on Effectiveness of Ultrasonic Hyperthermia

\section{KaCzmarek Katarzyna, JózefczAK Arkadiusz}

Institute of Acoustics, Faculty of Physics

Adam Mickiewicz University

Umultowska 85, 61-614 Poznań, Poland

Ultrasonic hyperthermia is a common medical procedure used supportively with anti - cancer therapies such as chemotherapy or radiotherapy. Due to absorption of ultrasonic energy by tissues heat in impact area is induced. This increase in temperature leads to some biological effects, such as increase of blood oxygenation or blood flow, which improves the anti-cancer treatment. During ultrasonic hyperthermia, some undesirable side effects e.g. pain, skin burn my appear due to high intensities of ultrasounds. Effectiveness of ultrasonic hyperthermia can be improved due to application of sonosensitizers - materials that lead to additional heat due to additional attenuation of ultrasounds. As such material, magnetic nanoparticles can be used. Experimental results have shown that due to application of magnetite particles into agar phantoms higher temperature increase can be obtain in the same time of sonication. From this it follows that through application of sonosensitizers we can achieve desirable increase in temperature in shorter time of treatment and also the effectiveness of ultrasonic hyperthermia can be improved without usage of high intensities.

\section{Acoustics-Related Aspects \\ of the Modernisation \\ of the Cracow Philharmonic Hall}

KAmisiński Tadeusz, Rubacha Jarosław Pilch Adam, BARuch Katarzyna

Department of Mechanics and Vibroacoustics AGH University of Science and Technology al. Mickiewicza 30, 30-059 Kraków, Poland

The historic building of the Cracow Philharmonic Hall was opened in 1930, providing for the multifunctional servility of the building. Currently, the 700 -seat auditorium is primarily used for symphonic concerts, while the conducted research and functional analyses have shown that an urgent modernization of the facility is needed with respect to the technical infrastructure and acoustics. In this paper, the selected solutions related to the interior acoustics and sound protection are proposed to improve the listening conditions in the auditorium. The presented simulation results involving the relevant acoustics-related parameters led to a new design of the audience, the closely positioned system of reflective panels, and the adjustable system of sound-absorbing curtains. In addition, the corresponding method to increase the acoustic insulation of the building facade was presented, which allows to deal with vibrations and noise generated by trams passing through the nearby street. Recommendations have been made to reduce the traffic involving heavy trams. Considering the historic nature of the building, the conservation requirements were an important factor influencing the proposed technical solutions.

$\star \star \star$

The Formula to the Evaluation of the Room Acoustics with Impedance Walls

\section{Kocan-KrawczyK Anna}

The Faculty of Electrical and Computer Engineering Department of Acoustics Rzeszow University of Technology al. Powstańców Warszawy 12, 35-959 Rzeszów, Poland

Based on the Fourier method, the room acoustics field is modelled. It is assumed the combination of uniform, impedance boundary conditions imposed on walls. Impedance boundary conditions are expressed by absorption coefficient values. All possible values, in discrete form, of the absorption coefficient are considered. An aim of the paper is to derive the formula to the description of the room acoustic field with impedance walls in full frequency range. The effectiveness of the impedance walls and the frequency is measured through the mean sound pressure level. The formula expresses the mean sound pressure level versus the absorption coefficient for separate frequencies and it is derived based on the least-squares approximation theory. This formula is a novelty in the room acoustics.

Theoretical considerations are illustrated, in the representative, rectangular cross section of the room, by quantitative results, which will be a point of reference to the numerical calculations. 


\section{Measurements of Insertion Loss of Mufflers with Microperforation for Single Cylindrical Duct Mode}

KOLBER Karolina

GorAzD Łukasz

SNAKOWska Anna

Faculty of Mechanical Engineering and Robotics AGH University of Science and Technology al. Mickiewicza 30, 30-059 Kraków, Poland

Construction of acoustic mufflers applying microperforated materials instead of porous materials leads to many substantial advantages, such as for example: resistance to environmental factors, dirt or fungus, which in turn eliminates cleaning issue impossible to achieve for porous materials. The aim of the paper is to present results of experimental investigations of noise reduction by filtering selected spinning modes present in the in-duct acoustic field. Spinning modes are especially strongly exited in ducts containing fans or other rotors. To investigate the reduction of noise the Insertion loss of some types of mufflers with microperforated panels mounted inside has been measured. The measurements have been carried on for generated selected single higher order modes and for different arrangements of the panels inside a cylindrical duct. The spinning mode modal filter was composed of four microperforated plates in the star shape located along the duct axis Measurements have been carried on for the plane wave and two consecutive spinning modes $(1,1)$ and $(2,1)$ excited separately by means of constructed by the authors mode synthesiser for Helmholtz number $k a=3.5$. The paper presents comparison between Insertion loss of mufflers with microperforated panels and mufflers of the same geometry but without any perforation indicating some noise reduction of any measured spinning mode. The experimental data are in good agreement with corresponding finite element method simulations.

$$
\star \star \star
$$

\section{Attenuation of Noise Through Owl's Wings}

\section{Kopania Joanna, BogusŁawski Grzegorz}

Lodz University of Technology

Piotrkowska 266, 90-924 Łódź, Poland

Noise can be defined as unwanted sound. There are many cases and applications that reducing noise level is of great importance, for example ventilation systems in vehicles and buildings, gas turbine intake/exhaust systems, aircraft engines or airfoils. Bionic is a novel approach to developing designs and products or to solving human problems by taking inspiration from nature. In this work, we focused on research specially crafted wings of the different species of owls - birds who are a good example of reducing aerodynamic noise. The attenuation bands of the wings were determined on a specially constructed test stand with an outlet to the anechoic chamber. The studies were done without flow. The pink noise as a source of sound was used. Frequency ranges were specified, in which the effect of sound attenuation was observed by the wings.

\section{Surface Acoustic Waves (SAW) - a Tool for the Study of Structural Changes in Ferronematics}

KopCANSKy Peter ${ }^{1}$, Tomasovicova Nataliia ${ }^{1}$ Gdovinova Veronika ${ }^{1}$, Majorosova Jozefina ${ }^{1}$ Timko Milan ${ }^{1}$, EBer Nandor ${ }^{2}$, Toth-Katona Tibor ${ }^{2}$ Bury Peter ${ }^{3}$, Vevericik Marek ${ }^{3}$, JADZYN Jan ${ }^{4}$

${ }^{1}$ Institute of Experimental Physics SAS

Kosice, Slovakia

${ }^{2}$ Institute for Solid State Physics and Optics

Wigner Research Centre for Physics

Hungarian Academy of Sciences

H-1525 Budapest, Hungary

${ }^{3}$ Department of Physics

University of Zilina

0100 Zilina 1, Slovakia

${ }^{4}$ Institute of Molecular Physics

Polish Academy of Sciences

Smoluchowskiego 17, 60-179 Poznań, Poland

About 5 decades ago the idea was born to mix nanosized magnetic particles (MPs) with nematic liquid crystals (LCs), in order to get fluids with a large magnetic susceptibility called ferronematics (FNs). In the presentation will be illustrated experimental data regarding structural changes so called Fredericksz transitions, response of these system to magnetic field especially to linear response in low magnetic field region, nematic-isotropic transition (TI-N) in presence of magnetic field as well as the idea how to prepare liquid ferromagnets based on FNs.

Acoustic methods are useful tool for the characterization of LCs, particularly their elastic and viscous parameters. Concerning the surface acoustic waves (SAW) they were used to determine the viscosity distribution in LC layer depending on applied electric field, as the SAWdriven LC light shutter or SAW sensor. Recently we have shown that the attenuation of SAW propagating along ferronematic liquid crystals is able to give information about structural changes in LCs, induced by both electric and magnetic fields. In this contribution, we present the utilization of SAW to study structural changes in ferronematic LC (6CHBT) doped with spherical, chain-like and rod-like magnetic nanoparticles induced by electric and weak magnetic fields.

$$
\star \star \star
$$

\section{Acoustic Images of the Seabed} in Selected Baltic Basins

KozaCzKa Eugeniusz ${ }^{1,2}$, Grelowska Grażyna ${ }^{1}$

${ }^{1}$ Faculty of Ocean Engineering and Ship Technology

Gdansk University of Technology

Narutowicza 11/12, 80-233 Gdańsk, Poland

${ }^{2}$ Polish Naval Academy

Śmidowicza 69, 81-103 Gdynia, Poland

Acoustic methods of underwater observation have been used since the beginning of the 20th century. At first, these were the navigation echosounders most often consisting of individual transducers. Then they began using sonar to detect dangerous underwater objects, the so-called forwardlooking sonar, which was mainly used in navy. In the second half of the twentieth century began construction of acoustic 
equipment; whose observation area was much larger than for the classic navigation or fishing echo sounder. Since then, the use of multi-beam echosounders and side-scan sonars has been observed. These devices allow the observation of significant areas of the bottom, which is essential especially in deep water studies. Observations are used to make accurate bathymetric maps of seas and oceans and to detect natural heterogeneities of sea bottom such as tectonic faults and artificial objects such as wrecks. At the turn of the 20th and 21st century, parametric multi-beam echosounders and parametric side-scan sonars were also introduced. The paper will present the acoustic images of the seabed of the Gulf of Gdańsk and the coast of the Baltic Sea. They allow you to provide up-to-date details of the seabed geometry.

\section{$\star \star \star$}

\section{Acoustics and Stage Technology Solutions at New Home for Polish National Radio Symphony Orchestra in Katowice (NOSPR)}

KozŁowski Piotr Z.

Chair of Acoustics and Multimedia

Faculty of Electronics

Wrocław University of Science and Technology

Wybrzeże Wyspiańskiego 27, 50-370 Wrocław, Poland

Paper concerns on widely understood acoustics and stage technology solutions adopted at new home for Polish National Radio Symphony Orchestra (Narodowa Orkiestra Symfoniczna Polskiego Radia, NOSPR - in Polish).

Challenges which were set for design team are presented at first part of publication. Technical assumptions accepted during concept process are described at second part of paper. Final acoustics and stage technology solutions present at design documentation which was used for building creation are discussed at third part of article. Noise control and sound proofing against external, internal and installations noises are discussed as well as topics concerns room acoustics at concert halls, rehearsal rooms and recording studio complex.

Difficulties observed during execution supervision process are listed. Acoustics user tune possibilities are described. Results of measurements done during acoustic tuning procedure are presented at the end of paper.

\section{The Influence of Structural \\ and Material Properties}

of Thermoacoustic Devices on Their Efficiency

Kruk Bartłomiej, DoBrucki Andrzej

Chair of Acoustics and Multimedia

Faculty of Electronics

Wrocław University of Science and Technology

Wybrzeże Wyspiańskiego 27, 50-370 Wrocław, Poland

Research in thermoacoustics began with the observation of heat transfer between an acoustic wave and a solid material. Using this interaction an intense sound wave could be applied to create engines as well as heat pumps. In spite of the absence of any mechanical parts, like pistons, energy conversion from heat flow into acoustic power flow is performed in the stack. The most important part of thermoacoustic devices is a regenerator or stack, where the process of converting acoustic energy into thermal energy, or vice versa, is taking place.

The aim of the paper is to present the properties of thermoacoustic prototypes (the impact of the material used to construct the regenerator and design parts of the acoustic system). This paper presents the results of the analysis of the materials used to construct the regenerator, and it describes constructed thermoacoustic devices. The authors of this research managed to achieve a $35^{\circ} \mathrm{C}$ temperature difference on the heat exchangers of the heat pump and create acoustic pressure level in the thermoacoustic engine reaching $183 \mathrm{~dB}$ SPL.

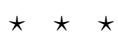

The Research on Impulse Rise Rapidity and Shift of Central Spectroid as Parameters for Differentiate Impulsive Noise Annoyance

\section{KUKULSKI Bartłomiej}

Department of Mechanics and Vibroacoustics AGH University of Science and Technology al. Mickiewicza 30, 30-059 Kraków, Poland

The paper presents the concept of similarity of features and parameters describing sound timbre - multidimensional attribute of auditory impression - to features and parameters describing impulsive noise annoyance. Features that influence sound timbre, such as energy distribution in the spectrum along with its change in time, rise time, its loudness or character, can be compared to impulsive noise, both in time domain (sudden increase in sound pressure, very short rise time and duration of the impulse) and frequency domain (flat spectrum over a wide frequency range along with its deformation over time). The research was performed on two parameters: impulse rise rapidity and shift of spectral centroid. An attempt was made to correlate values of these parameters with noise descriptors: peak, maximum and sound exposure levels, on the basis of which an impulsive adjustment is made according to ISO 1996.

\section{Program for Fast Prediction} of Window Acoustic Insulation

KwAPISZ Leszek ${ }^{1}$, JAKUBOWsKI Piotr ${ }^{1}$ KuśMIREK Magdalena ${ }^{2}$

${ }^{1}$ Institute of Fluid-Flow Machinery Polish Academy of Sciences Fiszera 14, 80-231 Gdańsk, Poland

${ }^{2}$ Environmental Laboratories Division Ship Design and Research Centre Szczecińska 65, 80-392 Gdańsk, Poland

The paper discusses the operation of the program, which predicts the acoustic insulation of a window in a wooden frame. The program is a tool that facilitates the window manufacturer:

a) fast estimation of its acoustic insulation,

b) quick fixes in the design to increase the acoustic insulation of the window, without the need for a prototype, 
which is especially important for short series made on special orders.

The basis of operation of the program is the experimental base for several dozen tested windows plus numerical results of calculations showing tendencies of changes of insulation when changing parameters of the window construction. The program is based on simplistic mathematical formulas approximating the results of experiments and numerical calculations. A general scheme of the program and performance results is presented.

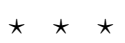

Study of the Sound Transmission Through the Window Using an Acoustic Camera and a Laser Doppler Vibrometer

KWAPISZ Leszek $^{1}$, JAKUBOWsKI Piotr ${ }^{1}$ KuśmireK Magdalena ${ }^{2}$, Kopaczewski Krzysztof ${ }^{2}$

${ }^{1}$ Institute of Fluid-Flow Machinery

Polish Academy of Sciences

Fiszera 14, 80-231 Gdańsk, Poland

${ }^{2}$ Environmental Laboratories Division

Ship Design and Research Centre

Szczecińska 65, 80-392 Gdańsk, Poland

The paper shows the results of the measurements of 3part window using an acoustic camera and a laser Doppler vibrometer (LDV). The results show that the acoustic camera and the vibrometer are complementary, highlighting other phenomena.

With LDV, the window acoustic insulation sensitivity to the inaccuracies of the frame fixing was confirmed. With the aid of an acoustic camera, it was confirmed the possibility of the micro gap appearance in the frame seal. The combination of both experimental methods becomes a good tool for detecting the imperfection of the construction and fixing of the window.

Analysis of the Influence of Temperature Change in Duct on the Acoustic Attenuation Performance of Selected Helicoidal Resonator

\section{ŁAPKA Wojciech}

Institute of Applied Mechanics

Poznań University of Technology

Skłodowskiej-Curie 5, 60-965, Poznań, Poland

The paper presents the analysis of the influence of temperature changes in cylindrical duct on the acoustic attenuation performance of selected helicoidal resonator. Changing the air temperature involves changing the length of the sound wave. When using resonators in the duct system to attenuate the unwanted sound, the change in air temperature will have a great impact on its performance. In the case of helicoidal resonators, active tuning technology has not yet been developed, so that even a small degree of resonance frequency can be adjusted to the frequency of the disturbance after the change of air temperature. Therefore, before the final version of such resonator is made it is necessary to analyze its effectiveness in a given installation, taking into account possible temperature fluctuations in the process, eg due to the change of the processed raw material.
The article discusses the important aspects of this subject using the practical experience as well as computer simulations, that uses the finite element method implemented in a specialized numerical environment called COMSOL Multiphysics-Acoustics Module.

Analysis of the Influence of Amplitude, Frequency and Phase Errors on Effectiveness of Noise Reduction of Multitone Signals by Active Noise Cancelling Systems

ŁUCZYŃski Michał

Chair of Acoustic and Multimedia

Wroclaw University of Science and Technology Wybrzeże Wyspiańskiego 27, 50-370 Wrocław, Poland

There are plenty of different configurations and algorithms of Active Noise Control Systems. Based on analysed papers and studies, it seems that among all systems of Active Noise Control the systems for cancelling multitone signals (e.g. engines, transformers) reach the highest efficiency in noise reduction. Analysis of the factors with the highest influence on attenuation efficiency is needed to pre-design estimate of effectiveness of noise reduction. The author of this paper conducts the analysis of limitations of the efficiency of active noise control systems. The theoretical analysis was carried out. Considered factors were amplitude, phase, and frequency errors. Calculations were performed for a plane, progressive wave propagated from two-point sound source. The obtained results were verified in measurements. The test conditions provide unidirectional propagation. The maximum of attenuation was found. Then, in the first step amplitude of a signal from secondary source was being changed. In the second step phase was being changed and then a frequency. The results of measurements confirmed theoretical calculation.

\section{Application of Grey Prediction Model GM(1,1) for Noise Exposure Forecasting}

\section{MAKAREwICz Grzegorz}

Institute of Radioelectronics and Multimedia Technology Warsaw University of Technology Nowowiejska 15, 00-665 Warszawa, Poland

Grey System Theory (GST) is increasingly used in various, often very distant fields. As the name implies, a grey system model refers to a system with incomplete and/or uncertain information. One of the advantages of GST is the ability to predict future values of the observation vector based on the small sample size (at least four) compared to other predicting methods. The most widely used forecasting model based on GST is the first order model of one variable designated as $\operatorname{GM}(1,1)$. The article describes the fundamentals and the rules for creating a $\operatorname{GM}(1,1)$ model and its use for forecasting noise exposure in working environment. Exemplary results of simulation calculations using the modified version of the so-called sliding forecast window are presented. 


\section{Shape Optimization of Rectangular Rooms for Improving Sound Quality at Low Frequencies}

Meissner Mirosław W., Zieliński Tomasz G.

Institute of Fundamental Technological Research

Polish Academy of Sciences

Department of Intelligent Technologies

Pawińskiego 5B, 02-106 Warsaw, Poland

A new method for the determination of optimum dimension ratios for small rectangular rooms has been presented. In a theoretical model, an exact description of the room impulse response was used. Based on the impulse response, a frequency response of a room was calculated to find changes in the pressure level over the frequency range 20-200 Hz. These changes depend on the source and receiver positions, thus, a new metric equivalent to an average frequency response was introduced to quantify the overall sound pressure variation within the room for a selected source position. A task of the numerical procedure was to seek a minimum value of a deviation of the pressure level response from a smooth fitted response determined by the quadratic polynomial regression. Numerical tests have shown that the best dimension ratios depend on the room volume and the sound damping in a room, and for small and medium volumes these ratios are roughly $1: 1.2: 1.45$, $1: 1.4: 1.9$ and $1: 1.5: 2.1$. When the room volume was suitably large, the ratio $1: 1.2: 1.44$ was found to be the best.

$$
\star \star \star
$$

The Methods and Instrumentation Used for Calibration of Sound Calibrators at the Central Office of Measures

\section{- National Metrology Institute in Poland}

MŁYŃska Aleksandra, Dobrowolska Danuta WiATER Mirosław

Central Office of Measures

Elektoralna 2, 00-139 Warszawa, Poland

The methods of calibration of sound calibrators as well as measurement systems used for calibration at the Central Office of Measures (GUM) are presented. There are two methods used. One of them is the comparison method, where the sound pressure level of the sound calibrator under test is determined using the calibrated class LS sound calibrator as a reference. The second one is the microphone method where sound pressure level generated by the sound calibrator under test is determined using a calibrated class LS or WS microphone and insert voltage technique. The methods of measurement of frequency and total distortion of the signal generated by sound calibrators are also described. Emphasis is placed on the new calibration system based on the Brüel \& Kjær PULSE System implemented recently at the GUM. The examples of results obtained for different sound calibrators both one-frequency and multifrequency as well as estimated measurement uncertainties are discussed. The problems occurring during calibration, especially concerning multi-frequency calibrators are presented.

\section{Acoustic Method as a Tool of Searching for New Applications of Ionic Liquids}

\section{Musią Małgorzata, Dzida Marzena}

University of Silesia, Institute of Chemistry

Szkolna 9, 40-006, Katowice, Poland

The subject of the present study are three ionic liquids with imidazole cation: 1-ethyl-3-methylimidazolium thiocyanate, $\left[\mathrm{C}_{2} \mathrm{C}_{1} \mathrm{im}\right][\mathrm{SCN}]$, 1-ethyl-3-methylimidazolium ethylsulfate, $\left[\mathrm{C}_{2} \mathrm{C}_{1} \mathrm{im}\right]\left[\mathrm{EtSO}_{4}\right]$ and 1,3-diethylimidazolium ethylsulfate, $\left[\mathrm{C}_{2} \mathrm{C}_{2} \mathrm{im}\right]\left[\mathrm{EtSO}_{4}\right]$. The speed of sound $(u)$ was measured at pressures up to $100 \mathrm{MPa}$ and in the temperature range from 293 to $318 \mathrm{~K}$. The isobaric heat capacity $\left(C_{p}\right)$ and density $(\rho)$ were measured at atmospheric pressure, in the temperature range from 293.15 to $318.15 \mathrm{~K}$ and from 288.15 to $363.15 \mathrm{~K}$, respectively. The thermodynamic properties of the tested ionic liquids at high pressures up to $100 \mathrm{MPa}$ and in the temperature range from 293 to $318 \mathrm{~K}$ were determined by the acoustic method, because this is one of the most reliable tool for determining $p \rho T$ data. The pressure and temperature effect on of the isothermal compressibility and the isobaric thermal expansion of the ionic liquids are discussed in the context of their potential application as hydraulic fluids.

\section{New Standards in Building Acoustics - State: June 2017}

\section{Nowicka Elżbieta, Iżewska Anna}

Building Research Institute

Filtrowa 1, 00-611 Warszawa, Poland

The basic aim of standardization in the field of building acoustics is to create the conditions to meet the basic requirement No. 5 "Noise protection" contained in the European Parliament Regulation 305/2011 on construction products and in the Polish Construction Law.

In order to properly standardize "noise protection" it is necessary to specify:

1) parameters, by which the acoustic properties of the products and structures and the acoustic conditions in the environment are determined and evaluated,

2) metric and computational methods used to determine acoustic parameters,

3) requirements for buildings and their surroundings.

The level of acoustic requirements for buildings and the external environment is determined by the individual countries and is quite varied depending on the economic possibilities. The evaluation parameters and methods of their designation are normalized at global or European level, within the framework of the work of the ISO/TC43/SC2 "Building Acoustics" and CEN/TC126 "Acoustic properties of building products and of buildings". Implementation of the EN/ISO norms for Polish standardization is dealt with by PKN Technical Committee No. 253 "Architectural Acoustics", which co-operates with the aforementioned. Committees.

The scope and status of standardization in the field of building acoustics is discussed in the paper. 


\section{Sound Damping of Impact Noise \\ in Floating Floors and Dynamic Stiffness of Resilient Layers}

\section{Nowotny Łukasz}

Building Research Institute

Filtrowa 1, 00-611 Warszawa, Poland

Frequent issue of present buildings is insufficient sound insulation of partitions including impact sound insulation. There are many ways to reduce impact sounds, in example: floor coverings, acoustic underlays and floating floors. Dynamic stiffness is the parameter used to describe acoustic properties of resilient materials used in floating floors. Dynamic stiffness is also the parameter used to calculate impact sound reduction index. Although, this method is not perfect. The relation between both parameters is not unequivocal. There is a need to precise the test method described in PN-EN 29052-1:2011 Acoustics - Determination of dynamic stiffness - Part 1: Materials used under floating floors in dwellings in a way that gives recurrent results.

This article discusses different factors that affect dynamic stiffness test results.

\section{$\star \star \star$}

\section{Algorithm of Multiparameter in vivo Breast Structure Imaging for Cancer Diagnosis \\ in Screening Tests with Ultrasound Tomography Method}

Opieliński Krzysztof J. ${ }^{1}$, PruChNiCKI Piotr ${ }^{1}$ JóźwIK Marcin ${ }^{2,3}$

${ }^{1}$ Chair of Acoustics and Multimedia

Faculty of Electronics

Wroclaw University of Science and Technology

Wybrzeże Wyspiańskiego 27, 50-370 Wrocław, Poland

${ }^{2}$ Chair of Gynaecology and Obstetrics

Faculty of Medical Sciences

University of Warmia and Mazury in Olsztyn

Niepodległości 44, 10-045 Olsztyn, Poland

${ }^{3}$ GYNEKA Center for Woman's Health

Łużycka 19, 30-658 Kraków, Poland

The paper presents the algorithm of multiparameter in vivo breast structure imaging, which performs the fusion of three different ultrasonic images and automatically determines areas of fat and glandular tissue and areas of benign and malignant tumour against the background of ultrasound scattering structures: vessels, milk ducts, ligaments, borders and fibers. The operation of the algorithm was verified by means of preliminary results of ultrasound tomography breast examination in vivo. The obtained results allow a prediction that the ultrasound tomography scanner implemented by a private investor in cooperation with the authors of this work offering fusion (assembling) of reconstructed images, may contribute to achieving a new standard for breast cancer diagnostics through fast and inexpensive screening tests.
Adaptation of Deep Learning Methods to Nocturnal Bird AUDIO Monitoring

PamuŁa Hanna ${ }^{1}$, KŁACZyŃski Maciej ${ }^{1}$ Remisiewicz Magdalena ${ }^{2}$, WszoŁeK Wiesław ${ }^{1}$ StOWELl Dan ${ }^{3}$

${ }^{1}$ Department of Mechanics and Vibroacoustics Faculty of Mechanical Engineering and Robotics AGH University of Science and Technology al. Mickiewicza 30, 30-059 Kraków, Poland

${ }^{2}$ Faculty of Biology, Bird Migration Research Station University of Gdańsk

Wita Stwosza 59, 80-308 Gdańsk, Poland

${ }^{3}$ Queen Mary University of London Centre for Digital Music, Machine Listening Lab Mile End Rd, London E1 4NS

Trends in the size of bird populations are good indicators of the general state of the environment. Different bird monitoring methods have been developed over the years, but surveys based on observations and bird-ringing programmes are the most common techniques. However, counting nocturnal migrant birds remains a particularly difficult task so other techniques must be applied. One possible method is automatic acoustic monitoring, which can supplement standard bird-monitoring schemes.

We investigated the automatic detection of migrant birds' night flight calls. Long-term recordings were collected during birds' autumn migration along the Baltic Sea coast. A deep learning method, convolutional neural network working on spectrograms, was adapted to detect the migrants' calls. The first results are promising (AUC $=$ $96.6 \%$ ), showing the potential of acoustic methods to supplement standard bird-monitoring techniques and to suggest directions for research.

$$
\star \star \star
$$

\section{Active Noise Control Methods} in Large-Scale Ventilation Systems

PAweŁczyk Marek ${ }^{1}$, Bismor Dariusz ${ }^{1}$, Czyż Krzysztof ${ }^{1}$ GŁówka Teresa ${ }^{1}$, Mościński Jerzy ${ }^{1}$, Szumski Maciej ${ }^{2}$ SzUMSKI Karol ${ }^{2}$

${ }^{1}$ Silesian University of Technology Institute of Automatic Control Krzywoustego 2, 44-100 Gliwice, Poland ${ }^{2}$ Plum, Ltd, Kleosin, Poland

The problem of noise in ventilation systems of large buildings is not new, but still unsolved. One can observe this problem while visiting offices, hotels, etc. The two main sources of noise in ventilation systems are: the ventilation unit's main fan, and the noise generated by turbulences induced by a ventilation duct air flow. This paper summarizes the results of a project on development of a passive-active noise control device dedicated to large-scale ventilation systems. The project resulted in creation of a prototype of the device with a very good noise reduction properties. Even application of only one such device in a large ventilation system allows to cancel noise efficiently. Better results can be obtained by installation of additional units near duct outlets. The device is currently being implemented in a few test buildings. 


\section{Impulse Response Measurements} in Niepolomice Forest

PieChowicz Janusz

ŻEGLEŃ Filip

Piechowicz Jakub

Department of Mechanics and Vibroacoustics

AGH University of Science and Technology

al. Mickiewicza 30, 30-059 Kraków, Poland

The reverberation in a forest arose as interference between the direct and ground-reflected sound and the scattering effects induced by trees and branches, the ground. The paper presents the results of impulse responses registered in various types of forest: in the forest with deciduous, coniferous and in a forest clearing. The experimental studies were performed in Niepolomice Forest. The portable measuring system used for testing made it possible to measure under field conditions. As results we obtained set of the parameters characterizing the reverb in the forest, i.e., reverberation time. The results show the differences in sound propagation in deciduous and coniferous forest, as well as in the forest "young" and "old", for which there are differences in the tree trunks perimeters and tree height. Based on the measurement sessions, a large number of recordings have been made, which will be used in multimedia work.

\section{Frequency Weighting in Sound Exposure Measurements Among Music Students}

Pietrzak Agnieszka Paula, JAsiński Maciej ŻERA Jan

Institute of Radioelectronics and Multimedia Technology Faculty of Electronics and Information Technology

Warsaw University of Technology

Nowowiejska 15, 00-665 Warszawa, Poland

The A-weighted pressure level is the most broadly used for noise measurements. However, there are studies, that suggest, that using the A-weighting curve can underestimate the influence, that low frequencies have on loudness and annoyance of sound. The A-weighting can produce inadequate conclusions regarding the risk of noise induced threshold shift. Among music students, during rehearsals, concerts and individual practicing, sound level often exceeds the limit of $85 \mathrm{~dB}(\mathrm{~A})$. This study compares the A-weighted measurements and measurements with no frequency response curve during orchestra and individual practicing of music students at The Fryderyk Chopin Music University in Warsaw to assess the amount of low frequency sound produced by various instruments.

\section{Multiobjective Optimization of Schroeder Diffusers} PILCH Adam

Department of Mechanics and Vibroacoustics AGH University of Science and Technology al. Mickiewicza 30, 30-059 Kraków, Poland

Sound phase diffusers (Schroeder diffusers) reflect sound evenly in many directions as a result of a flat spectrum of depth sequence. Optimization helps to improve diffuser parameters in some case even twice, comparing to usually used quadratic residuum sequences. Nowadays in diffusers optimization only directional diffusion coefficient is considered, while other important parameters are not controlled.

In the paper, relationships between diffusion, scattering and absorption coefficients are presented. Results of calculations for different combinations of parameters are presented. Diffusers constructed on multiobjective optimized sequences, characterized by high sound diffuser coefficient together with low sound absorption coefficient, what is especially important for structures used in large volume auditoriums.

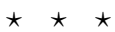

\section{Database Containing HRTF Measurements Results in XML Format}

Plaskota Przemysław, Stasiak Jakub

Chair of Acoustics and Multimedia

Wrocław University of Science and Technology

Wybrzeże Wyspiańskiego 27, 50-370 Wrocław, Poland

In recent years, interest in Head Related Transfer Functions (HRTF) has grown, also due to the possibility of automated measurements. Automation makes it possible to perform high spatial resolution measurements for many subjects in short time. Different research centers publish measurement results in a data format that is not standardized. As a result, the reuse of measurement results by other researchers is limited.

The paper presents an XML format database proposal that unifies the presentation of HRTF measurement results. A review of the published databases was made. On this basis tools for converting selected databases to the proposed format were presented. The database also includes a HRTF measurement results for 52 subjects. HRTF was measured using a system for the rapid and automatic system.

$$
\star \star \star
$$

Effectiveness of the Inverse Multi-Quadratic RBF in an Acoustic Indoor Problem

\section{PrędKa Edyta, Brański Adam}

Department of Acoustics

Faculty of Electrical and Computer Engineering

Rzeszów University of Technology

al. Powstańców Warszawy 12, 35-959 Rzeszów, Poland

The nonsingular meshless method is analysed in an acoustic indoor boundary problem. The solution is assumed in the form of series of radial bases functions. These bases constitute the inverse multi-quadratic functions. The aim of the paper is an analysis of the effectiveness of these functions in the description of the room acoustic. The acoustic boundary problem with uniform, impedance walls is considered. Impedance boundary conditions are expressed by absorption coefficient values. All approximate results of numerical calculations are compared to the exact solution and to the approximate solution obtained based on Hardy's multi-quadratic functions. It is appear, that tested bases functions may be used to the approximate description of the indoor acoustic. 


\section{Characterisation of the Probability Distribution of the Long-Term Noise Indicators in Continious Monitoring of the Gdańsk City}

Przysucha Bartosz ${ }^{1}$, Pawlik Pawel ${ }^{2}$ MACiEJCZYK Jerzy ${ }^{3}$, WszołeK Tadeusz ${ }^{2}$ SzElĄG Agata ${ }^{4}$

${ }^{1}$ Department of Quantitative Methods and Management Lublin Univeristy of Technology

Nadbystrzycka 38, 20-816 Lublin, Poland

2 Department of Mechanics and Vibroacoustic

AGH University of Science and Technology

al. Mickiewicza 30, 30-059 Kraków, Poland

${ }^{3}$ League Against Nois

Bernardyńska 2/61, 02-904 Warszawa, Poland

${ }^{4}$ Institute of Structural Mechanics

Cracow University of Technology

Warszawska 24, 31-155 Kraków, Poland

During the estimation of long-term noise indicators $L_{D}$, $L_{W}, L_{N}$ it is essential to determine the uncertainty of these indicators. Typically, the uncertainty is given in the form of a $95 \%$ confidence interval. Such ranges are estimated based on the probability distribution of the feature from which the population is derived. When a variable has a normal distribution, it can be assumed that the centered mean has a t-student distribution. Further, based on this statistic it is possible to build a confidence interval. Having a sufficiently large measurement test, one can take advantage of the Lindeberg-Levy theorem and assign the centered mean normal distribution.

In measurement samples of noise indicators, asymmetries of probability distributions are often observed. It affects the way in which the uncertainty intervals are constructed. In general, the intervals may be asymmetric. An important aspect of the interval estimation process is the knowledge of probability distributions of long-term noise indicators.

The article presents probabilistic analyses and identification of probability distributions of long-term noise indicators based on data from the continuous monitoring of the city of Gdańsk.

\section{$\star \star \star$}

Tonal Noise in the Working Environment

- Research Review and Possibilities of Assessment in the Aspects of Annoyance

RAdosz Jan

REJMAN Małgorzata

Central Institute for Labour Protection

- National Research Institute

Czerniakowska 16, 00-701 Warszawa, Poland

Noise as a stressor can contribute to the development of a variety of illnesses (eg. hypertension, peptic ulcer, neurosis), distract attention, hinder work, and reduce performance. Building equipment such as heating, ventilation and air conditioning systems, as well as office equipment computers, printers and telecommunications equipment - can emit tones as a result of rotating parts such as fans, pumps, etc. The widespread use of these devices in the working environment translates potentially up to several hundred thousand jobs in Poland. Numerous literature reports indicate that the annoyance of tonal noise is greater than broadband noise. In the assessment of working environment there are no criteria for tonal noise. This paper presents the most common methods for determining tonal noise based on Tone-to-Noise Ratio, Prominence Ratio, Tonal Audibility and Aures' Tonality Model. The work also discusses the possibility of carrying out an assessment of tonal noise in the aspect of annoyance.

$$
\star \star \star
$$

Analysis of Acoustic Emission Signal Generated in Mechanically Loaded Reinforced and Non-Reinforced Concrete Specimens

RANACHOWSKI Zbigniew ${ }^{1}$, LEWANDOWsKI Marcin ${ }^{1}$ Schabowicz Krzysztof ${ }^{2}$, Logoń Dominik ${ }^{2}$

${ }^{1}$ Institute of Fundamental Technological Research Polish Academy of Sciences

Pawińskiego 5B, 02-106 Warsaw, Poland

${ }^{2}$ Faculty of Civil Engineering

Wrocław University of Technology

Wybrzeże Wyspiańskiego 27, 50-370 Wrocław, Poland

In the paper, a new method aimed to classify Acoustic Emission (AE) sources operating simultaneously with different spectral characteristics. The method employs the partitioning the entire signal sample collection into small subsections. The simplified discrete spectral characteristic of dominating waveform within the subsection is determined. Averaged spectral characteristics are then applied to learn the dedicated neural network arrangement. The neural network is finally used to classify the waveforms within the investigated large signal collections. The efficiency of the procedure was tested applying the records of AE signal derived during mechanical tests of four types of concrete specimens.

$$
\star \star \star
$$

The Acoustic Radiation Impedance of a Vibrating Circular Plates Including the Effect of Antiresonance

\section{RDZANEK Witold J.}

RDZANEK Wojciech P.

Department of Mechatronics and Control Science Faculty of Mathematics and Natural Sciences University of Rzeszów

Pigonia 1, 35-310 Rzeszów, Poland

The effect of the antiresonance on the radiation acoustic impedance of a clamped circular plate embedded into a flat rigid baffle has been considered in this study. The excitation has been assumed to be homogeneous on the central part of the plate's surface. The attenuation from the fluid and the internal damping of the plate have been neglected. Consequently, simplified expressions have been obtained and the use of the modal analysis was not necessary. This made it possible to emphasize the effect of the antiresonance. The numerical analysis of the obtained results has been presented. 


\section{Sound Radiation of a Spherical Cap on a Circular Sphere in the Outlet of a Finite Semi-Spherical Rigid Cavity \\ RDZANEK Wojciech P. \\ Department of Mechatronics and Control Science Faculty of Mathematics and Natural Sciences \\ University of Rzeszów \\ Pigonia 1, 35-310 Rzeszów, Poland}

A rigorous analysis of sound radiation by a pulsating sphere forming a resonator together with a lower hemispherical cavity of a finite thickness is presented. The problem is solved by dividing the entire region into the three subregions. First of them surrounding the sphere and containing the cavity, second containing the annular upper hemi-sphere, and the third containing the remaining space. The continuity conditions are applied to obtain the acoustic pressure in all the subregions. The acoustic field is calculated both in the near-field and in the far-field. The acoustic radiation impedance is calculated using the impedance approach. The resonance frequencies are determined, for which a significant growth of the sound pressure level is observed as well as the sound field directivity. The effect of the thickness of the rigid hemi-sphere is examined. The accuracy and convergence of the rigorous results is tested numerically.

\section{$\star \star \star$}

Development, Relevance and Perspectives

of Engineering Acoustics between Physical Science and Applied Technology

SCHEUREN Joachim

Müller-BBM GmbH

Planegg/Munich, Germany

It was in the second half of the last century only that acoustics, being underestimated by lacking challenges then, was realigned by the requirements of fast reconstruction and corresponding rapid mechanization. At that time, engineering acoustics promptly became relevant because efficient methods of construction and production had to cope with growing quality requirements. Since then engineering acoustics has developed from an occasionally involved special discipline to an indispensable interdisciplinary field of modern technology.

The paper demonstrates this development together with the resulting technical performance of engineering acoustics by means of exemplary case studies in noise control and sound design. With particular respect to road tyre noise control and active car interior sound design, the iterative cycle of research, development and application will highlight the mutual influence of physical science and applied technology. The conceptive impact of modelling rolling noise generation, the great potential of consequent application of acoustic principles to silent road surface design and the technological influence on realistic perspectives of innovative active sound control show how science and technology have to interact to preserve and further develop efficient interdisciplinary engineering acoustics and thus to enable technical progress with both, growing comfort and environmental compatibility.
The Adaptive Vibration Control of Circular Plate - Comparison Tests with FIXeD CONTROL

SierżĘGa Mariusz, Leniowska Lucyna

Department of Mechatronics and Control Engineering University of Rzeszów

al. Rejtana 16c, 35-959 Rzeszów, Poland

Article presents adaptive vibration control method of circular plate based on online identification. The article concerns the problem of a design, development and experimental verification of an active feedback vibration control system of circular plate. The plate vibrations were excited by a loudspeaker located inside the cast iron cylinder. Vibrations of the plate are measured using noncontact method - laser vibrometer, that ensures high precision of measurements. Vibrometer signal in the feedback loop was proportional to the vibration velocity in orthogonal direction to the surface of the test plate. The control input was applied to the plate by a MFC disk, attached to the plate in its centre. Control rules has been designed using pole placement method and OE parametric model. The mathematical model of the object was identified online using the RRLS algorithm. Designed controller has been implemented and tested on a real-time platform - PowerDAQ with xPC Target environment. The article presents the results of implementation and performed comparison tests for the adaptive and fixed controllers.

\section{Duct Acoustics - Results of Theoretical and Experimental Research}

SNakowska Anna

Faculty of Mechanical Engineering and Robotics AGH University of Science and Technology al. Mickiewicza 30, 30-059 Kraków, Poland

Duct acoustic is the branch of acoustics concerned with the sound propagation in waveguides and pipes and is of substantial interest in many areas of acoustics and noise control because of numerous technical applications. The problems encountered within the duct acoustics are considered accounting for some specific features of the system such as geometry, properties of the duct walls, range of frequencies of the sound field or flow of the medium. The most frequently discussed type of duct is the infinite or semi-infinite hard duct with circular cross section. Those types of ducts are of particular interest as components of heating, ventilation and air-conditioning systems (HVAC), aircraft jet and automobile engines, and others. The frequency $\omega$ of the sound wave and the duct radius a determine shape of the field and play the main role in the in-duct phenomena and so the parameter called reduced frequency (or Helmholtz number) $k a=\omega a / c$ was introduced to simplify the field description. With increase of $k a$ the sound field in duct becomes more complicated, the propagating wave is no longer the plane wave but is multimodal, each of the modes being a certain solution of the Helmholtz equation. If the presence of the so-called higher modes is neglected the results obtained are referred in literature as "the low-frequency approximation". Nevertheless, analysing the phenomena in jet engine or large compressor ducts the multimodal approach is inevitable and is of great 
concern in contemporary duct acoustics. Mathematically, a sound wave in a duct can be briefly described by means of eigenfunctions of the Helmholtz equation. Their orthogonality property fairly simplifies some considerations and leads to introducing new techniques of the sound analysis, such as the mode matching method or the mode decomposition method. Both of these methods are powerful tools to carry on the advance field analysis such as transmission and reflection of modes at the duct outlet, directivity of the field radiated from an unflanged duct, power emitted outside and radiation impedance, impedance matrix along the duct and many others which will be addressed in the paper applying the solution of the diffraction problem of an unflanged circular duct.

\section{$\star \star \star$}

\section{Analysis of Uncertainty of Measurement of Insulating Insulation of Air Conditioning Instruments}

\section{Stryczniewicz Tytus, WszoŁek Tadeusz}

Department of Mechanics and Vibroacoustics AGH University of Science and Technology al. Mickiewicza 30, 30-059 Kraków, Poland

Acoustic protection of buildings is one of the biggest challenges faced by architects and designers. One of the key aspects is the adequate sound insulation of the building partitions and its measurement verification. The quality of the result obtained and the factors affecting it are determined by analysing the uncertainty of measurement. Factors affecting the uncertainty of the final measurement result include partial uncertainty of sound pressure level measurement, time of acoustic absorption determination, and geometric parameters of the barrier. The aim of this paper is to present an analysis of the uncertainty of measuring the insulation from airborne sounds with particular emphasis on its components and their effect on total uncertainty. The analysis of the uncertainty was carried out on the examples of measurement of the insulation of the intermedia walls and of the outer partitions in residential buildings in Cracow. In the examples and statistical analysis, the factors that have the greatest impact on the end result of the measurement and the quantitative share in the total uncertainty have been identified.

\section{Dimensional Analysis and Similarity Criteria in Model Studies of Sound Transmission Through Building Partitions}

SzeląG Agata, BARuch Katarzyna, Flaga Andrzej

Department of Mechanics and Vibroacoustics

AGH University of Science and Technology

al. Mickiewicza 30, 30-059 Kraków, Poland

In the field of acoustics, model studies are conducted to a very limited extent due to the lack of strictly defined criteria of similarity between the real system and its scale model. The paper presents a number of theoretical, numerical, and experimental analyses, performed to determine the appropriate criterion numbers, mainly for use in the issues of sound transmission through the building partitions. Based on the obtained results, it will be possible to carry out acoustic insulation measurements on a scale that will significantly reduce the cost and availability of this type of research. Also new types of analyses will be available, such as studies of sound transmission throughout the building based on its scale model.

$$
\star \star \star
$$

\section{Preliminary Experimental Investigations of the SOUND Radiation by a Clamped Vibrating Circular Plate}

Szemela Krzysztof, RDzaneK Wojciech, ŻYŁKa Wojciech

Department of Mechatronics and Control Science

Faculty of Mathematics and Natural Sciences

The University of Rzeszów

Pigonia 1, 35-310 Rzeszów, Poland

This study is focused on an experimental investigations related to a vibroacoustic behaviour of a clamped circular plate embedded in a flat rigid baffle. The analysed object has been placed inside a semi-anechoic chamber. The plate has been excited by means of a point force with an asymmetric location. The measured physical quantities, the vibration velocity and the sound pressure, have been normalized by the value of exciting force amplitude. This approach allows the obtained results to not be dependent on the excitation's parameters. Making use of a laser vibrometer, the amplitude of normalized vibration velocity has been measured for the plate's and baffle's surface. The influence of the plate vibrations on the baffle vibrations has been investigated and consequently, the perfectly rigid baffle assumption has been verified. The sound pressure has been measured at fixed field points. The amplitude of normalized sound pressure has been analysed as a function of the vibration frequency as well as the angular variables of the spherical coordinate system. The determined from measurements resonance frequencies have been compared with those theoretically calculated.

\section{Application of ADC and ADP Descriptors} to Identify the Acoustic Emission Signal Generated by the Partial Discharges

\section{within Selected Frequency Bands}

Szerszeń Grzegorz ${ }^{1}$, Witos Franciszek ${ }^{2}$

${ }^{1}$ Department of Electronics and Telecommunications State Higher Vocational School in Tarnów

Mickiewicza 8, 33-100 Tarnów, Poland

${ }^{2}$ Department of Optoelectronics

Faculty of Electrical Engineering

Silesian University of Technology

Krzywoustego 2, 44-100 Gliwice, Poland

The research results of partial discharge in the systems with modeled sources, carried out by calibrated acoustic emission method. are presented.

The research has been carried out on measurement stand consisting of a vat of oil with mounted modelled sources, the test set for generating high voltage, author measurement system 8AE-PD (acoustic emission method) and system TE571 (electric method).

Recorded signals were filtered in selected frequency bands, and then for the filtered signals, the following char- 
acteristics were calculated: phase-time characteristics, averaged phase characteristics, the frequency characteristics and averaged Short-Time Fourier Transform spectrograms.

Based on the calculated characteristics there were performed qualitative analysis of selected recorded signals and quantitative analysis for selected signal parameters in the time domain and descriptors with acronyms ADP and ADC calculated in the field of the threshold of discrimination as well. Analysis showed the possibility of distinguishing of signals generated by partial discharge and noise. Description of signals using of ADP and ADC descriptors showed additional capabilities to confirm the presence partial discharge sources within the examined object.

$$
\star \star \star
$$

Simulation of Measurements and Reconstruction of Images by the Ultrasound Method of Continuous Ultrasonic Wave Tomography in Circular Geometry

Świetlik Tomasz, OpIeliński Krzysztof J.

Chair of Acoustic and Multimedia, Faculty of Electronics Wrocław University of Science and Technology Wybrzeże Wyspiańskiego 27, 50-370 Wrocław, Poland

Modern Doppler tomography (DT) method, also known as ultrasound continuous wave tomography (CWUT) is one of the ways to use the continuous ultrasound wave and Doppler effect to visualize the internal structure of objects scattered ultrasonic waves and submerged in water. The signal necessary to perform an image reconstruction in CWUT is the so-called Doppler signal, recorded around an object with an internal structure. This method can be used in medical diagnostics in vivo. In order to analyse the possibilities of imaging using the CWUT in the circular geometry, a computerized method of Doppler signal determination was developed, assuming that scattering ultrasonic wave from infinitely small objects is uniformly in all directions. The Doppler frequency determination algorithm and the high-speed tomography image reconstruction algorithm were also developed. On the basis of the obtained results the analysis and the discussion of the possibilities and limitations of the DT method was done.

\section{Examinations of the Degree of Atherosclerosis by Means of Input Vascular Impedance}

TRAwiński Zbigniew ${ }^{1}$, Hilgertner Lech ${ }^{2}$

${ }^{1}$ Institute of Fundamental Technological Research

Polish Academy of Sciences

Department of Ultrasound

Pawińskiego 5B, 02-106 Warsaw, Poland

${ }^{2}$ Department of General and Thoarcic Surgery

Warsaw Medical University

Banacha 1a, Warszawa, Poland

The input vascular impedance was determined using ultrasound continuous wave Doppler flow meter and a system for tracking arterial wall movements. The study was conducted in the control group (30 volunteers) and in four groups (10 patients in each group). Patients were grouped according to the criteria of atherosclerotic stage, which was the degree of artery stenosis: $20-49 \%, 50-69 \%, 70-$ $89 \%, 90-99 \%$ and occlusion, based on ultrasound or duplex tests. The results of the Student's t-test showed the statistical significance of the separation of longitudinal resistance Ro results from the control group and sequentially four groups with a defined degree of atherosclerotic carotid artery at a $95 \%$ confidence level with significance level from $p<5.2 \mathrm{E}-3$ for $20-49 \%$ - for the receiver operating curve (ROC), the area under curve $(\mathrm{AUC})=0.76, p<8.6 \mathrm{E}-9$ for the stenosis $50-69 \%$ - for the ROC curve AUC $=0.913$, $p<6.3 \mathrm{E}-9-$ for the stenosis $70-89 \%$ for ROC curve AUC $=0.94$, to $p<8.1 \mathrm{E}-10$ for stenosis above $90 \%$ and occlusion, for ROC curve AUC $=0.97$. After combining data for patients with internal carotid artery stenosis from $50-89 \%$ the results of the Student's $t$-test showed the statistical significance of the separation of the longitudinal resistance results Ro derived from the control group and the combined group with a confidence level of $95 \%$ at significance level $p<2.8 \mathrm{E}-10$, for ROC curve AUC $=0.942$.

\section{Spherical Arrays Applied in Room Acoustics}

\section{VORLÄNDER Michael}

Institute of Technical Acoustics, RWTH Aachen University 52056 Aachen, Germany

Multi-channel spherical loudspeakers became popular for control of directional radiation. They have been introduced in shapes of cubes, dodecahedra, or higher-order discrete representations of spheres. In this contribution, a spherical source with a partial Gaussian distribution of 28 channels is presented. With sequential measurements and rotation of the sphere, a radiation of effectively 23 rd order of spherical harmonics is obtained, as long as the acoustic conditions are time-invariant. In auralization, this source can reproduce musical instruments, for example, for more realistic analysis of room reflections. Filter design and various applications are discussed as well as an approach for measurement of binaural room impulse responses reciprocally on the one hand, and the combination of spherical loudspeaker arrays with spherical microphone arrays in a multiple-input-multiple output, MIMO system, on the other.

$$
\star \star \star
$$

\section{Optimization of Viola Da Gamba Soundboard Designed from 3D Printing Material}

\section{Wilczyński Tomasz J., Filipek Roman}

AGH University of Science and Technology al. Mickiewicza 30, 30-059 Kraków, Poland

The article is describing the whole modeling process of viola da gamba soundboard. The most frequently used material for soundboards is spruce. It has been replaced by a composite material that enables using the $3 \mathrm{D}$ printing technology. Different material parameters require also different values of the soundboard parameters to maintain the characteristic timbre of the pattern instrument. For this purpose, a multi-objective optimization has been made which based on the computer model of soundboard created using FEM. A computer experiment was planned and carried out using an optimal space filling algorithm (OSF) in 
space for the three input parameters (soundboard thickness at the edge and center, side plate thickness), and four output parameters (two modal frequencies and corresponding MAC values). They have been taken into account within the cost function. Response surfaces has been determined by the universal kriging method. The optimization process of model parameters, realized by screening method, allowed to choose their values describing shape definitely thicker then wooden plate. The designed plate of viola da gamba has two modal frequencies with difference less than $9 \%$ from pattern. Moreover, a MAC coefficient describing similarity of forms is higher than 0.7 .

\section{$\star \star \star$}

\section{Side Scan Sonar as Tool for Imaging the Seabed of the Gdańsk Bay}

\section{Witos-Okrasińska Dominika}

Gdańsk University of Technology

Narutowicza 11/12, 80-233 Gdańsk, Poland

The main purpose of the article is to present the impact of environmental conditions on the accuracy of seabed imaging using hydroacoustic systems. Natural factors, especially sea surface ripples and heterogeneous distribution of sound velocity in water, have an effect on the change in the sound path. Although in both cases the mechanism of phenomena is completely different, they play a decisive role in the mapping of the bottom and underwater objects. The paper discusses the influence of both factors: wave and refraction caused by heterogeneous sound velocity distribution on the accuracy of the bottom projection by the side sonar. All these phenomena cause marine tests to be subject to various errors. Their analysis can help you get more accurate results.

The reflections on the influence of environmental conditions and, in particular, the velocity distribution on the accuracy of underwater images, will be illustrated by the results of measurements in the Gdańsk Bay area between 2016 and 2017 and 2000-2010 multiannual studies.

\section{$\star \star \star$}

\section{The Absorptive Dummy Head for Binaural Recordings}

\section{Wrzeciono Piotr}

Faculty of Applied Informatics and Mathematics

Warsaw University of Life Sciences

Nowoursynowska 159, 02-776 Warszawa, Poland

The paper presents the realization of dummy head model made by using an absorptive element and two microphones with the cardioid characteristics. The absorber was made from the polyester fiber material with thickness $14 \mathrm{~cm}$. The proper acoustic isolation between microphones and the modification of their direction characteristics were obtained by the use of the mentioned absorber. The test recordings and the listening tests were made to evaluate the subjective perception of spatial hearing. During this tests, three kinds of headphones were used: open, semiopen and closed. $95 \%$ of the listeners correctly positioned the apparent sound sources. This result is very similar to the other obtained by testing the commercial solutions and presented findings can lead to construction of absorptive dummy head which use can be more cost-effective compering with the most popular present solutions.

\section{The Influence of Different Kinds of Externally Generated Noise on Tinnitus Perception}

WrzoseK Małgorzata

Department of Logic and Cognitive Science Institute of Psychology, Adam Mickiewicz University Umultowska 85, 61-614 Poznan, Poland

Objective: The goal of this work was to investigate the influence of different kinds of externally generated noise on tinnitus perception. Psychophysical Experiments: 1) Notched noise was used to test whether the continuous Zwicker tone illusion may resemble tonal tinnitus. 2) The relationship between the psychometric tuning curves and the tinnitus tuning curves was investigated. 3) The effectiveness of amplitude modulated sounds in producing the residual inhibition was tested. Results: 1) The illusion resembled experience of tonal tinnitus, and in some cases made it inaudible. 2) The characteristics associated with the perception of tinnitus and the perception of real tone were very different. 3) Amplitude modulated sounds were found to be more effective than unmodulated white noise in eliciting the temporal elimination of tinnitus perception. Conclusions: Generally, the results confirm the usability of externally generated noise in research and work with tinnitus patients. Noise can be applied diagnostically and therapeutically.

$$
\star \star \star
$$

\section{Vibroacoustic Parameters in the Assessment of Stutterers}

\author{
WszoŁek Wiesław ${ }^{1}$, Malczyk Grażyna ${ }^{2}$ \\ ${ }^{1}$ Department of Mechanics and Vibroacoustics \\ AGH University of Science and Technology \\ al. Mickiewicza 30, 30-059 Kraków, Poland \\ 2 "Perfetto" \\ Wiedeńska 88, 30-147 Kraków, Poland
}

Speech is one of many ways of communicating information. it is an intellectual process. The intentional transmission of information is essential for communicating with the environment. It affects the emotions of both the broadcaster and the receiver, as well as the whole course of interpersonal and social communication. In the case of people stuttering communication is very difficult, sometimes even impossible. Stuttering is a disorder of speaking fluency, occurring both in children and in adults. Stuttering makes it impossible to move smoothly from one articulation to the other, as well as maintaining proper tempo, rhythm and intonation of speech. This disorder is related to disintegration or discoordination of all movements of the voice channel taking part in speech. This disorder prevents a free, smooth transition from one articulation to another. The paper presents the research methodology, the results of which will get to know the phenomenon of stuttering in the voice channel (larynx), it also supports the process of 
rehabilitation of people stuttering. The results of a study on explaining the origination of the stuttering process were also presented. Using vibroacoustical imaging of the process of creating and blocking sound in people stuttering.

\section{Tuning Mechanism in the Model of Central Auditory Processing for Pitch Perception}

\section{ZięBAKOWSKI Tadeusz}

Institute of Manufacturing Engineering West Pomeranian University of Technology Al. Piastów 17, 70-310 Szczecin, Poland

One of the main tasks of the hearing process is to improve the quality of spectral analysis which is initialized on the ear level. This effect is usually called a tuning and it is observed both physiologically and psychologically. The mechanism of tuning, however, is unclear so far. The paper proposes a central processing model, in which the tuning mechanism is described as an iterative process, in which psychophysical spectrum is estimated on the base of spectral-time signal generated by ear. It is assumed that the central mechanisms control processes on peripheral level by efferent neurons and outer hair cells. In the modeling of the peripheral part of the auditory system, the model developed by Meddis and Hewitt was partially used.

\section{Application of Artificial Genomes to Animal Vocal} Communication Structure Modelling

ZuBER Krzysztof W.

OpIELIŃsKI Krzysztof J.

Chair of Acoustics and Multimedia

Faculty of Electronics

Wrocław University of Science and Technology

Wybrzeże Wyspiańskiego 27, 50-370 Wrocław, Poland

The article presents the application of a simulated genome method to modelling of non-deterministic, pattern based structures of animal vocal communication. The proposed method, where analogously to the DNA both the data and the instructions for its manipulation are encoded and processed in a single string, is based on the functioning of DNA, which enables for data variability, polymorphism, and adaptive and nondeterministic expression. Thanks to these characteristics, the method can elastically model phenomena which are structured, yet random to a degree, such as animal vocal communication. The method's capability for modelling the syntax and grammar of animal signals is presented on two examples: the vocal communication of blue whales and Bengalese finches, and the results show the usefulness of the method.

$$
\star \star \star
$$

\section{An Attempt to Validate the Proposed Method of Simulation of the Sound Generation Process in Blown Idiophones}

\section{ŻŁOBIŃSKI Bartosz}

Warsaw University of Technology Plac Politechniki 1, 00-661 Warszawa, Poland

Blown idiophones are musical instruments like the accordion, the harmonium, the harmonica and the reed organ pipes, in which a thin bar with its own stiffness serves as the vibrator, a stream of air as the actuator and the resonator can take various forms. It was proposed previously to simulate the process of sound generation in these instruments numerically, using the Finite Volume Method for solving the coupled problem of a flow in a fluid, a displacement of a solid and a propagation of an acoustic wave. The need of performing a validation was remarked. In the article, an attempt to validate the proposed method was described. It involves a construction of a physical model. The data from measurements serve as reference values. Some theoretical and practical difficulties was encountered and the appropriate solutions was proposed. Partial results, obtained despite problems, was introduced and likewise suggestions for the further research. 